\title{
Una invitación poco común: hablemos purépecha
}

\author{
Frida Villavicencio
}

CLAUDINE CHAMOREAU, 2003

Parlons purépecha. Une langue du Mexique

L' Harmattan, París-Budapest-Turín.

$\mathrm{N}$ o obstante que el purépecha $o$ tarasco ${ }^{1}$ es una de las lenguas indígenas de México relativamente mejor documentadas y que ha sido objeto de estudio desde los primeros años de la colonia hasta nuestros días, los textos que nos brindan una introducción general de esta lengua son escasos. La mayoría de los trabajos que se han escrito sobre el purépecha en las últimas décadas tratan de manera específica algún aspecto de su estructura (la fonología, la morfología, el léxico) o se circunscriben a un fenómeno particular (los sufijos espaciales, las marcas de voz, el sistema de caso). El panorama es aún más desolador si consideramos que la mayor parte de estos textos están dirigidos a un grupo restringido de lectores constituido por especialistas y que resultan, por lo tanto, poco accesibles a públicos amplios.

FRIDA VILLAVICENCIO: CIESAS-México.

Desacatos, núm. 15-16, otoño-invierno 2004, pp. 239-243.

${ }^{1}$ Una de las primeras preguntas que se hace cualquier persona que se acerca por primera vez al estudio de esta lengua es, precisamente, sobre el nombre tanto de la lengua como del grupo que la habla. Este tema constituye un punto polémico que aún no se ha podido resolver del todo. Para un panorama general de algunas de las implicaciones ideológicas que conlleva esta discusión véase Frida Villavicencio "La denominación de un pueblo. Una relación entre lenguaje y poder” en Pedro Márquez (coord.), ¿Tarascos o purépechas?, en prensa.

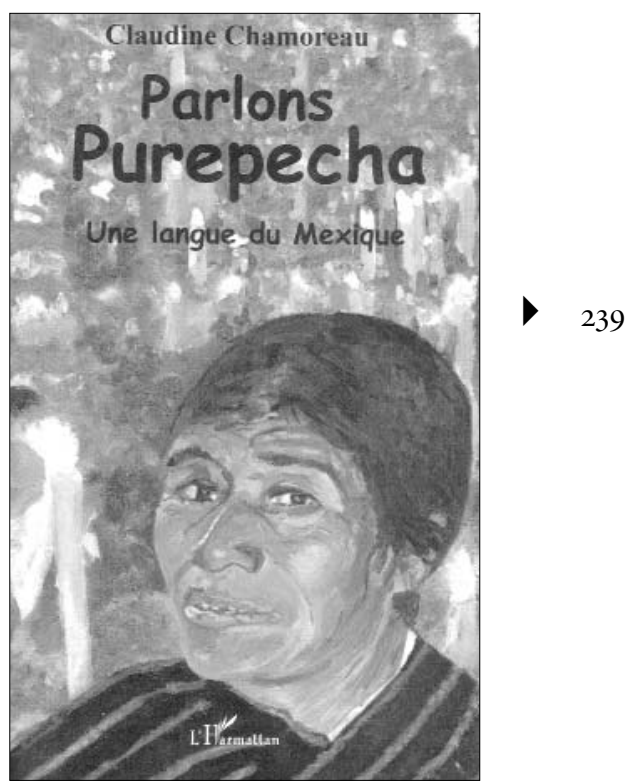

En este contexto, Parlons purépecha viene a llenar un vacío hasta ahora existente. Con un lenguaje claro, sencillo y ameno, su autora, Claudine Chamoreau, nos introduce a las complejidades de esta singular lengua y nos acerca a la historia y a la cultura del pueblo purépecha. Sus observaciones se basan principalmente en el habla de Jarácuaro, comunidad que 
alguna vez fue una isla y que actualmente, como consecuencia de la desecación experimentada por el lago de Pátzcuaro, se ha unido a tierra firme constituyendo de esta manera una península.

Parlons purépecha se inscribe en una tradición que se interesa en las lenguas en tanto manifestaciones concretas de una actividad humana universal realizada por individuos particulares que son miembros de comunidades históricas. El interés de la autora es dar cuenta de una lengua real, viva, en constante cambio; para lograrlo, Chamoreau ha optado por presentar la lengua enmarcada por el contexto histórico y cultural en el cual se desarrolla, lo que explica la estructura que ha elegido para integrar este libro. Además de una breve introducción en la impulsan a emprender esta obra y diserta sobre la denominación de la lengua y la cultura, el libro se organiza en seis capítulos:

En el primero, "Historia y territorio", la autora presenta una descripción general del territorio en el que actualmente se habla el purépecha, proporciona algunos indicadores demográficos que caracterizan a la población hablante y realiza una breve semblanza de la historia de este pueblo. Este capítulo constituye un marco de referencia que permite al lector considerar los tópicos recurrentes en los estudios purepechistas, sobre los que se ha escrito mucho y se ha concluido poco: la incierta filiación lingüística del idioma, el origen histórico de la cultura, la dinámica socioeconómica actual, entre otros. Nos enteramos

entonces que el purépecha es una de las más de cincuenta lenguas indígenas de México cuyos hablantes se estiman actualmente en poco más de 100 mil personas que habitan principalmente al noroeste del estado de Michoacán, en lo que se conoce cono la región purépecha. Dicha zona comprende hoy una veintena de municipios en los cuales se asientan más de cien comunidades reconocidas como purépechas. Sabemos de la reducción que ha experimentado el territorio desde la época del contacto hasta nuestros días, así como el incierto origen del grupo y de su lengua. Tenemos noticia de las fuentes históricas más conocidas que guardan lo poco que se sabe de este grupo antes de la llegada de los españoles, aunque ambos fueron escritos en la época colonial (la Relación de Michoacán y el Lienzo de Jucutacato), así como de los últimos aportes de la arqueología al conocimiento del grupo y su cultura. Para finalizar este capítulo, Chamoreau nos habla de la vitalidad de este pueblo y de su inserción en la sociedad nacional, pondera la dinámica de su desarrollo, que se manifiesta a través de las actividades económicas que realizan, la organización tradicional que conservan y el fenómeno de la migración que los afecta.

Los siguientes tres capítulos conforman el núcleo propiamente lingüístico del texto. Si bien cada uno de ellos tiene un peso diferente, que va de más a menos, en conjunto nos proporcionan un panorama general de la lengua. El denso segundo capítulo, "La gramática", condensa la mayor parte de la información sobre la estructura del purépecha que se ofrece en este libro. A través de él, Chamoreau nos lleva desde la fonología hasta la oración compleja, pasando por las distintas clases de palabra existentes, la morfología tanto del sustantivo como del verbo, sin dejar de tocar brevemente algunos de los procesos sintácticos más comunes y la estructura de la oración. Sabemos entonces que el purépecha es una lengua altamente verbalizadora, exclusivamente sufijante, de orden flexible, casual y pospositiva.

El tercer capítulo, "La formación del vocabulario", presenta un panorama general del amplio campo que atañe a la formación de palabras en esta lengua. En él se tratan temas como la derivación, la composición y los préstamos. Sabemos entonces que de piréni (cantar) se deriva pireri (cantor) y pirékwa (canción); que de karáni (escribir) se deriva karári (escritor) y karákata (lo escrito); y que de tarhéni (arar, labrar) se deriva tarhékwa (arado), tarhéri (labrador, campesino) y tarhéta (sementera, milpa). Chamoreau hace especial énfasis en los locativos y los direccionales tan idiosincrásicos a esta lengua y entonces nos enteramos que para expresar dolor en purépecha, debemos especificar la parte del cuerpo que nos duele:

p’améarhani si lo que nos duele es el estómago, pero p'améchani si lo que nos duele es la garganta, p'amék'urhani si son las manos, p'améts'ïni si es la cabeza y p'aménduni si son los pies. También nos percatamos que en esta lengua los direccionales tienen una gran importancia, a tal punto que si decimos ch'anápani indicamos que 
alguien se aleja (del hablante) jugando, pero que si decimos chianápuni indicamos que alguien se acerca (al hablante) jugando. En un contexto de contacto asimétrico, como el que caracteriza el desarrollo de esta lengua desde hace ya casi cinco siglos, no podía faltar el tema de los préstamos. En este apartado Chamoreau se ocupa tanto de los términos españoles que han pasado a formar parte del léxico purépecha como: wáka (vaca), amyu (amigo), sábadu (sábado), pasárini (pasar), etc., como de aquellos vocablos originalmente purépechas que se han integrado al léxico del español regional e incluso del español de México, entre ellos: charal, huarache, curinda, chacuaco, pirékua, tepache, timbiriche, lista a la que yo agregaría tacuche, vocablo que ahora ha caído un tanto en desuso pero que tuvo una amplia difusión en la época de los "pachucos".

En el cuarto capítulo, "La conversación cotidiana", la autora ofrece el recuento de algunas expresiones y palabras de uso común en una interacción verbal: diversas formas de saludo y despedida y algunas preguntas de ubicación así como de información. No obstante que se trata del capítulo más breve, los ejemplos concretos de uso de la lengua que en él se proporcionan permiten al lector apreciar algunas características pragmáticas del purépecha. Podemos apreciar entonces que existe una forma familiar: ¿nari erantiski? para decir "Buenos días / ¿cómo amaneciste?", y una forma respetuosa de saludar: ¿nats'ï erántiski? para decir "Buenos días / ¿cómo amaneció usted?" Y que la palabra para

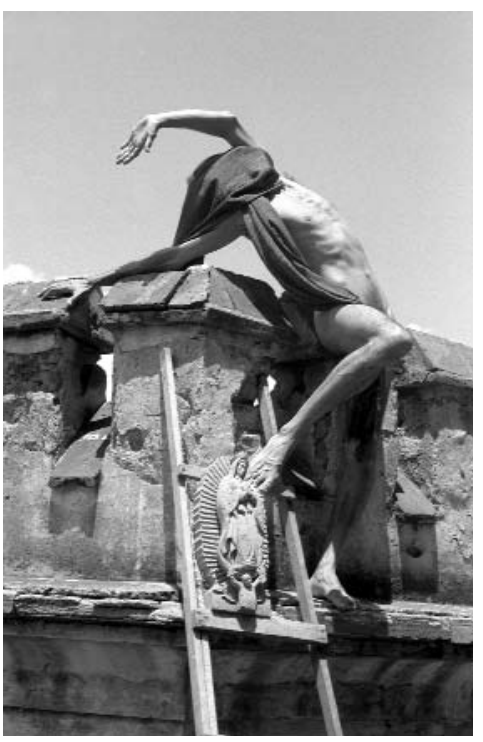

Virgen / Foto: Lilian Stein.

dar las gracias: diosï meyamukwa, procede de la expresión diosï meyámu (dios se lo pague), muy probablemente acuñada en el siglo XVI por los frailes evangelizadores.

Todo aquel que lo haya intentado estará de acuerdo en que dar cuenta de manera breve, clara y precisa de una lengua natural, cualquiera que ésta sea, es un esfuerzo titánico. La complejidad del objeto a describir es tal que parecería que no importa por donde decidamos empezar, la lengua "se nos viene encima". No por ello Chamoreau se ha amedrentado y en Parlons purépecha propone un camino bastante tradicional que procede por niveles. El recorrido inicia con la fonología, discurre por la morfología flexiva y algunos aspectos básicos de sintaxis, la formación de palabras y termina en la pragmática. Tal vez la parte más compleja de la tríada que conforman los capítulos dos, tres y cuatro sea el capítulo sobre gramática en el cual es difícil establecer un hilo conductor claro. La autora realiza la presentación a partir del reconocimiento de clases de palabras (sustantivo, adjetivo, verbo, adverbio), pero también a partir de funciones; por ejemplo, lo que ella llama personales (personnels) incluye palabras, clíticos y sufijos (los pronombres personales, los clíticos pronominales de sujeto y de objeto y los sufijos verbales de beneficiario). La morfología nominal flexiva se trata sólo después de haber presentado todos los posibles modificadores de la frase nominal, tal vez - aunque la autora no entra en detalles- porque las categorías de número y de caso funcionan básicamente en el nivel de frase y no son atribución exclusiva del sustantivo. En la sección que trata sobre las marcas de caso habla de $m p u$ como el elemento más polisémico del sistema y sólo más tarde reconoce que se trata de la forma gramaticalizada de la posposición jimpó. ${ }^{2}$

La terminología utilizada también merecería una nota, Chamoreau es tal vez la purepechóloga que más se aparta de cierta tradición ya establecida; si bien, a diferencia de la práctica que ha seguido en sus publicaciones dirigidas a especialistas, en Parlons purépecha la autora adopta una ortografía práctica, ${ }^{3}$ continúa utilizando la terminología

\footnotetext{
2 Curiosamente, Mary Foster hizo lo mismo en The Tarascan Language, University of California Press, Berkeley, 1969 (University of California Publications in Linguistics, 56).

3 Se trata de la ortografía propuesta por María Guadalupe Hernández Dimas y Fernando Nava, en Jánhaskapani. Juchari anapu jimpo, Uárhi-FAPROP, Morelia, Michoacán, 2000
} 


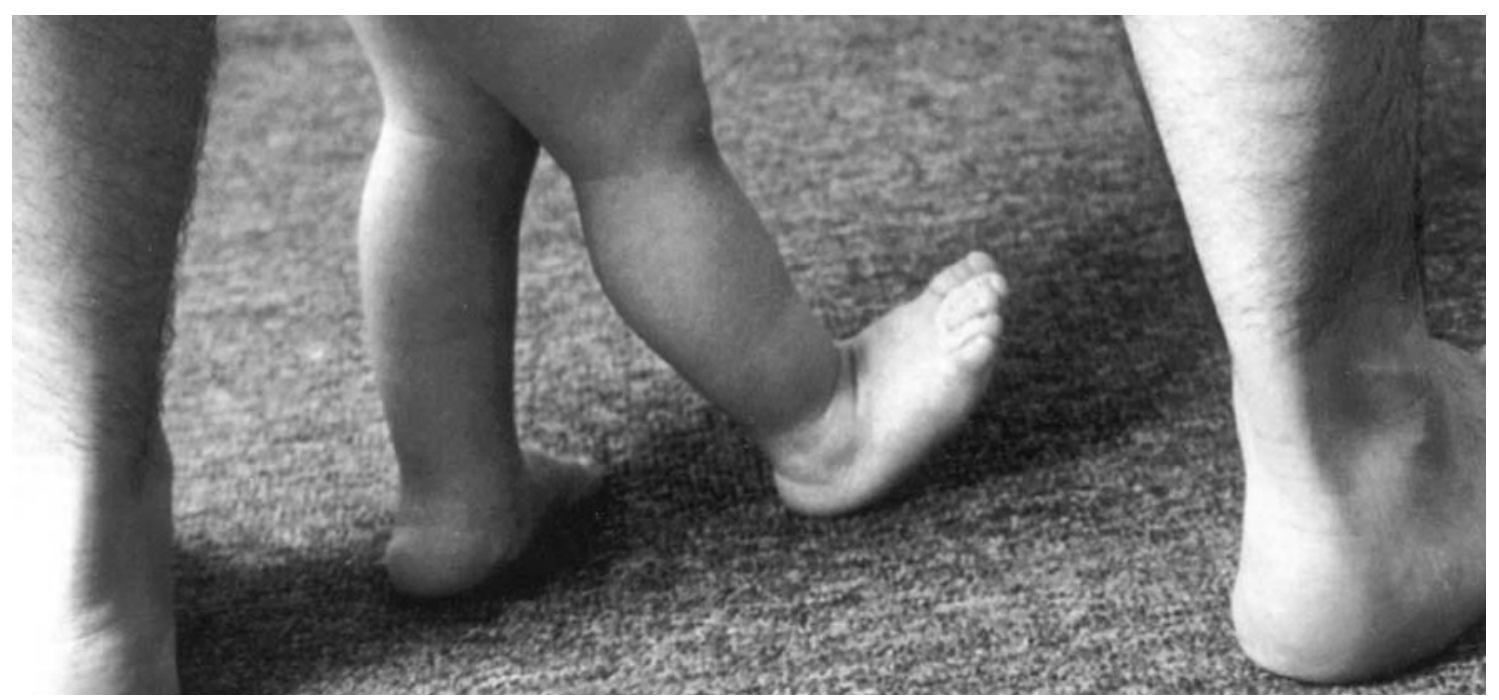

Paternidad afectiva. Calendario Coriac 2001 / Foto: Tania Robledo. Cortesía de Coriac.

que la caracteriza sin hacer ningún comentario o advertencia al respecto. El lector se encuentra con términos tales como indices personales que refieren a lo que la mayoría de los autores contemporáneos reconocen como clíticos de persona, habla de un aspecto aoristo que en otros estudios corresponde a un perfectivo ${ }^{4} \mathrm{y}$ un modo asertivo en donde otros autores reconocen un indicativo, etcétera.

En el quinto capítulo, "Los hablantes y el surgimiento de la escritura", Chamoreau se propone introducirnos a la cultura purépecha mediante algunas manifestaciones concretas de la

${ }^{4}$ Chamoreau ha argumentado a favor del reconocimiento de un aoristo en "Le système verbal du p'urhépecha”, Systèmes verbaux, BCILL, 1998, pp. 55-69, y en Description $d u$ purépecha parlé sur des îles du lac de Patzcuaro (Mexique), 3 tomos, Université Paris

V-René Descartes, Groupe des Sciences Humaines-Sorbonne, París, 1998 [tesis de doctorado]. amplia tradición oral de este pueblo. En él toca temas que tienen que ver con aspectos sociolingüísticos como: la relativa variación dialectal existente en el área, la educación bilingüe y las políticas lingüísticas que se han implementado en el área, así como el papel que actualmente desempeñan algunos medios masivos de comunicación como la radio indigenista y la prensa ${ }^{5}$ en el proceso de rescate, revitalización y

\footnotetext{
${ }^{5}$ La radio indigenista ha desempeñado un significativo papel en el área purépecha durante los 25 años de su presencia. Son las jóvenes generaciones de investigadores quienes empiezan a interesarse por sus complejidades. Véase Ivonne Grethel Chávez, El papel de la radio indigenista en el municipio de Cherán, Michoacán, CIESAS, México, 2003 (tesis de maestría); Nelly Calderón de la Barca, El caso de la XEPUR, "la voz de los p’urhépechas", Centro de Estudios de las Tradiciones, El Colegio de Michoacán, 2001 (ensayo terminal de maestría). El estudio de la presencia y el papel que ha tenido la prensa tarasca y en los medios de comunicación en general en sus distintas modalidades y en sus diversas épocas está aún por realizarse.
}

difusión de la lengua y la cultura de este pueblo.

Con el afán de contextualizar el uso del purépecha, la autora brinda ejemplos del papel central que la lengua desempeña en ámbitos centrales a la cultura como la música, la cocina, la toponimia y algunas de las ceremonias del calendario ritual. Conocemos así la importantísima y singular tradición de cantar pirékwas, expresión viva de la vena poética y musical que caracteriza a este pueblo; nos deleitamos con una receta de atápakwa, un plato tradicional de la cocina regional; nos enteramos que nombres como Querétaro, Pátzcuaro y Zacapu son de origen purépecha y recreamos algunas de sus ceremonias más significativas como el día de muertos y el recién creado (y recreado) "Año Nuevo Purépecha".

Por último Chamoreu nos ofrece un vocabulario elemental del purépecha en el que se recoge, en versión bilingüe 
francés-purépecha y purépecha-francés, una buena muestra del léxico actual. Las entradas se presentan en orden alfabético lo que permite una consulta fácil. No obstante su reducida extensión, el vocabulario de Parlons purépecha se viene a sumar a las cinco obras lexicográficas contemporáneas disponibles para esta lengua; 6 su importancia radica en que éste registra el habla de Jarácuaro y, con ello, una muestra del complejo mosaico de variantes existentes en la región. ${ }^{7} \mathrm{Tal}$ vez porque el texto está dirigido, en primera instancia, a un público internacional, la autora ha sentido la necesidad de introducir al final del vocabulario un glosario de aquellos términos del español mexicano y regional que pueden requerir de una explicación puntual para aquellos lectores no familiarizados con la cultura mexicana, se incluyen aquí términos como: atole, chapata, comal, pozole, tamal, nixtamal, etc., que en una versión destinada al público mexicano tal vez no sería tan necesario.

Si bien se trata de un texto introductorio, concebido para un público

${ }^{6}$ Paul de Wolf, "Vocabulario", en Curso básico del tarasco hablado, El Colegio de Michoacán-Gobierno del estado de Michoacán, Morelia, 1991. Maxwel Lathrop, Vocabulario del idioma tarasco, Literatura tarasca, Cherán, Michoacán, 1973. Alberto Medina Pérez, Vocabulario p’urhépecha-español, Morelia, 1997. Pablo Velásquez Gallardo, Diccionario p'urhépecha, FCE, México, 1978. Juan Velázquez et al., Vocabulario práctico bilingüe p’urhépechaespañol, INEA-Michoacán, Michoacán, 1997. ${ }^{7}$ Chamoreau proporciona otro vocabulario basado en el habla de esta comunidad, esta vez en versión purépecha-español en Purépecha de Jarácuaro, El Colegio de México, México, 1993 (Archivo de Lenguas Indígenas de México). amplio no necesariamente especializado, dada la variedad de los temas que en él se consideran y la amplitud de la información —especialmente lingüística- que se maneja, hubiera sido útil que en algún momento la autora advirtiera al lector el poco conocimiento que aún se tiene sobre diversos aspectos de la lengua y la cultura purépecha. Temas que actualmente siguen en debate o en espera de estudios específicos son, por ejemplo: la determinación del orden básico de la lengua, las clases de palabra que deben ser reconocidas, la tipología de las oraciones relativas, la posible caracterización del purépecha como una lengua de voz media, el reconocimiento o no de procesos de composición, la nomenclatura de los diversos tiempos y aspectos, etcétera.

Lo mismo vale para temas tales como la denominación del grupo y su lengua, la estandarización y la normalización ortográfica, o el debate sobre las diversas variantes que se deben o pueden reconocer (tema, por cierto, en el que Chamoreau se interesa ahora). El fenómeno de la migración y sus repercusiones en la cultura y la lengua, así como el doble papel que los migrantes y sus remesas desempeñan: por una parte en la conservación de las tradiciones culturales y, por la otra, en la transformación de las comunidades y su organización. El papel que los medios masivos de comunicación tienen en la revitalización de la lengua y la proliferación de estaciones de radio independientes, periódicos, sitios web y revistas electrónicas editados por hablantes. Los resultados de más de medio siglo de educación bilingüe, el surgimiento de la normal indígena y el proyecto de una universidad intercultural en la zona, etc. Una bibliografía más amplia que remitiera al interesado a lecturas específicas y especializadas sobre cada uno de los distintos temas y que ubicara al lector no sólo en el campo de la lengua y la cultura purépecha sino también en el amplio, complejo y dinámico campo de los estudios purepechistas hubiera podido ser de gran ayuda.

Como su nombre lo sugiere, Parlons purépecha es una invitación a acercarnos no sólo a esta lengua sino también a los seres humanos que la hablan, seres humanos que como todos nosotros, son producto de una historia y portadores de una cultura. En este sentido, se trata de un trabajo que hacía mucha falta en una época en la que desafortunadamente no abundan los textos introductorios y de difusión que, como éste, se sustenten en un conocimiento profundo del tema que tratan. Se suele olvidar que para que las lenguas indígenas persistan necesitan de hablantes vivos que las recreen y las actualicen e investigadores comprometidos no sólo con las lenguas que estudian sino también con los pueblos que las hablan. Una versión de Parlons purépecha destinada al público de habla hispana sería muy útil tanto para los mismos hablantes de las lenguas que están ávidos de materiales sencillos pero serios que se ocupen de su lengua y de su cultura como para el resto de la sociedad mexicana que podría entonces empezar a conocer un poco más de la riqueza que en este campo encierra un país como México. 